\title{
Hut-Like Pillar Si Solar Cells
}

\author{
Arti Agrawal ${ }^{* 1}$, Francisco J. Cabrera-España ${ }^{1}$, Andrew Oates $^{2}$ and Hari Reehal ${ }^{2}$ \\ 1. School of Mathematical, Computer Science and Engineering, City University London, London, EC1V OHB, United Kingdom \\ 2. School of Engineering, London South Bank University, 103 Borough Road, London, SE1 OAA, United Kingdom \\ *arti.agrawal.1@city.ac.uk \\ Abstract: This paper introduces an exciting new hut-like texturing pattern that shows lower \\ Reflectance than pyramids and nanowires in the shorter and longer wavelengths respectively.

\section{Introduction} \\ OCIS codes: (040.5350) Photovoltaic; (350.6050) Solar energy.
}

A large number of investigations have focused on lowering the Reflectance of solar cells by different means. The techniques being used generally lead to either high costs [1] or high surface recombination and high surface-tovolume ratio $[4,6]$. The motivation of this work is the search of a new texturing pattern capable of giving low Reflectance with low surface-to-volume ratio. In the next section, we describe the benefits of the hut-like pattern.

\section{Pattern details and comparison with other patterns}

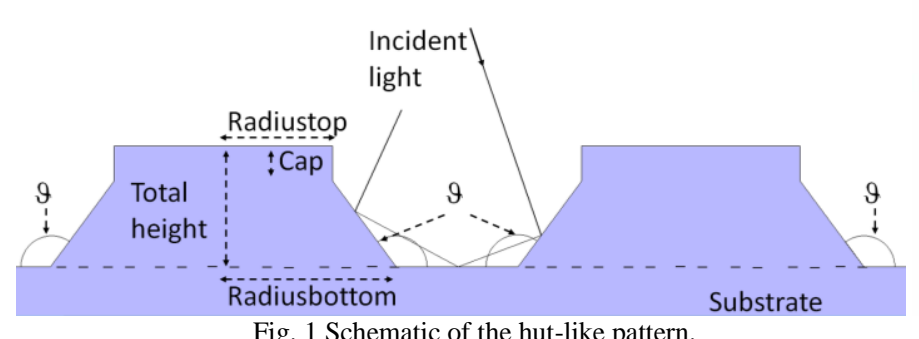

Fig. 1 Schematic of the hut-like pattern.

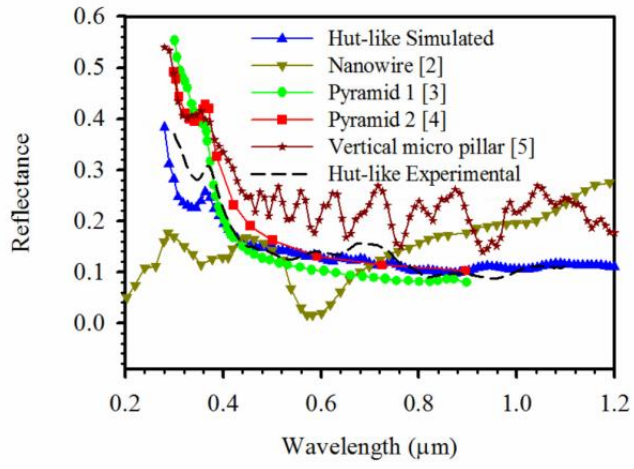

Fig. 2 Comparison between texturing patterns.

In Fig. 1 we show a schematic of the hut-like pattern where the parameters Radiustop, Radiusbottom, Cap and Total height represent the radius on the top of the hut, radius of the top and bottom of the hut respectively, height and Cap.

The shape of the pattern enables an efficient light trapping in the area in-between huts. The incident light penetrating in-between huts reflects multiple times against the side walls of the hut which are tilted with an angle $\vartheta$. As this angle varies, the number of multiple reflections, may increase or decrease. We need the maximum number of multiple reflections to enhance the chances of absorption for any given Surface Coverage (i.e. hut concentration).

In Fig. 2 we compare the hut-like (simulated and experimental) with other patterns with similar dimensions: nanowire, pyramid and vertical micro pillar [2-5]. The parameters of "hut-like simulated" are: total height $=1 \mu \mathrm{m}$, Cap $=0.2 \mu \mathrm{m}$, Radiustop $=0.5 \mu \mathrm{m}$ and $\vartheta=115^{\circ}$. The Reflectance from the vertical micro pillar is higher than the hutlike for the entire spectrum. When compared with nanowires and pyramids, the Reflectance from the hut-like is lower on longer and shorter wavelengths respectively. The difference in the performance is because the area inbetween the huts allows better optimization of the multiple reflections of the incident light; enhancing the chances of light absorption. The parameters of "hut-like experimental" are: total height $=0.99 \mu \mathrm{m}$, Cap $=0.0 \mu \mathrm{m}$, Radiustop= $0.316 \mu \mathrm{m}$ and $\vartheta=115^{\circ}$. The experimental results of the hut-like corroborate those from simulations. Furthermore, there is a good match in almost the entire spectrum, between the experimental and simulated results.
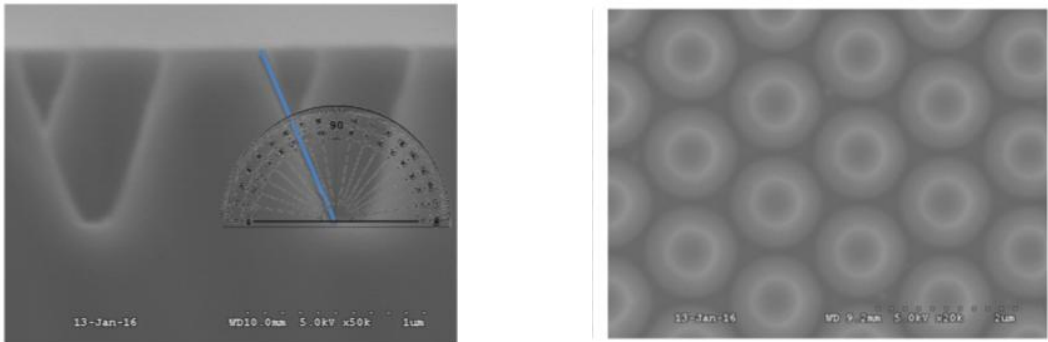

Fig. 3 a. Cross-section SEM image after 20 mins etching. b. Planar SEM image after 20 mins etching

In Figs. $3 \mathrm{a}$ and $\mathrm{b}$ we show a SEM crossection and top view respectively of the sample with the hut-like pattern. The pattern was lithographically defined on a $\mathrm{Si}(100)$ wafer by the technique of Displacement Talbot Lithography 
using a EULITHA PhableR 100 system [7]. A lift-off process was used to create a regular array of 10-nm-thick chrome discs for use as an etch mask in plasma etch process. An SF6/C4F8 plasma was used to create the tapered $\mathrm{Si}$ rods using the following parameters: $20 \mathrm{~W}$ RIE, $1200 \mathrm{~W}$ ICP, $5 / 55 \mathrm{sccm}$ gas ratio, pressure $10 \mathrm{mTorr}$ [8]. These parameters gave an etch rate of $\sim 50 \mathrm{~nm} / \mathrm{min}$ with a DC bias of $\sim 100 \mathrm{~V}$. We will report further experimental results of the hut-like pattern and compare them with simulations.

\section{Simulation results on parametric analysis}
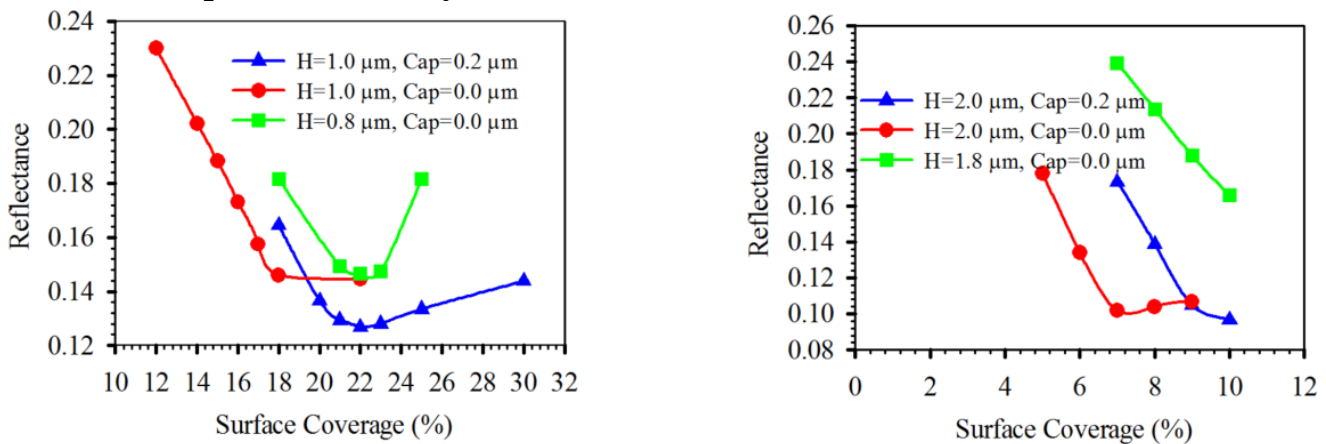

Fig. 4 a. Performance comparison changing total height by means of changing Cap for total height $=1 \mu \mathrm{m}$. b. Performance comparison changing total height by means of changing Cap for total height $=2 \mu \mathrm{m}$.

In Figs. $4 \mathrm{a}$ and $\mathrm{b}$ we discuss the results obtained via simulations using Lumerical FDTD [9]. We compare the Reflectance as total height varies by changing Cap as function of Surface Coverage, while keeping other parameters constant. In Fig. 4 a the Reflectance from the pattern with total height $=1 \mu \mathrm{m}$ and $\mathrm{Cap}=0.2 \mu \mathrm{m}$ is the lowest among the three lines. However, the small difference between the lines indicates that the vast majority of the light is absorbed at the slope part of the hut. Nevertheless, some small part of the light is absorbed by Cap. In Fig. $4 \mathrm{~b}$ we see similarly trend since the lowest Reflectance is given when total height $=2 \mu \mathrm{m}$ with Cap $=0.2 \mu \mathrm{m}$. In this graph the attention goes to, the difference on the performance between the two cases with same total height. The very small difference, between these cases, indicates that as the total height increases, more of the light is absorbed by the sloped part of the hut and less by Cap. Therefore, in the case of Cap reducing to zero, making easier the fabrication, would not affect much the performance as the sample fabricated by our collaborators at University of Bath.

\section{Conclusions}

The hut-like pattern offers lower Reflectance than nanowire and pyramids with similar dimensions and it can be fabricated with good accuracy. As total height increases, the influence of Cap on the performance reduces which suggests a low impact on performance when Cap $=0.0 \mu \mathrm{m}$.

\section{Acknowledgements}

Sample preparation in the Nanofabrication Facility at the University of Bath was supported by the EPSRC 'Access to Nanolithography' programme (grant ref. EP/K040324/1).

\section{References}

[1] Yan, X., Poxson, D. J., Cho, J., Welser, R. E., Sood, A. K., Kim, J. K., Schubert, E. F., "Enhanced omnidirectional photovoltaic performance of solar cells using multiple-discrete-layer tailored-and low-refractive index anti-reflection coatings," Adv. Funct. Mater. 23, 583-590 (2013).

[2] Wang, J., Li, Z., Singh, N., Lee, S., "Highly-ordered vertical Si nanowire/nanowall decorated solar cells," Opt. Express, 19, 23078-23084 (2011).

[3] Hou, Y., Abrams, B. L., Vesborg, P. C. K., Bjorketunm, M. E., Herbst, K., Bech, L., Seger, B., Pedersen, T., Hansen, O., Rossmeisl, J., Dahl, S., Norskov, J. K., Chorkendorff, I., "Photoelectrocatalysis and electrocatalysis on silicon electrodes decorated with cubane-like clusters," J. of Photonics for Energy, 2, 026001 (2012).

[4] Papet, P., Nichiporuk, O., Kaminski, A., Rozier, Y, Kraiem, J., Lelievre, J. F., Chaumartin, A., Fave, A., Lemiti, M., "Pyramidal texturing of silicon solar cell with TMAH chemical anisotropic etching," Solar Energy Materials and Solar Cells, 90, 2319-2338 (2006).

[5] Cabrera-Espana, F. J., Rahman, B. M. A., Agrawal, A., "Study of optical properties of textured Si solar cell with micro pillars," In Title of Proceeding, (Optical wave and waveguide theory and numerical modeling workshop (OWTNM), Nize, 2014).

[6] Dan, Y. Seo, K., Takei, K., Meza, J. H., Javey, Al., Crozier, K. B., "Dramatic Reduction of Surface Recombination by in Situ Surface Passivation of Silicon Nanowires," Nano Lett. 11, 2527-2532 (2011).

[7] Solak, H.,Dais, C., Clube, F., "Displacement Talbot lithography: a new method for high-resolution patterning of large areas," Optics Express, 19, 10686-10691 (2011).

[8] Saffih, F., Celal, C., Alanoud, A., Mustafa, Y., Bo, C., " Fabrication of silicon nanostructures with large taper angle by reactive ion etching," Journal of Vacuum Science \& Technology, B 32, 06FI04 (2014).

[9] Lumerical Solutions, Inc., (http://www.lumerical.com) (2003-2016). 